\title{
The Cryptochrome1 (cry1) Gene has Oscillating Expression Under Short and Long Photoperiods in Sesamia nonagrioides
}

\author{
Anna Kourti ${ }^{*}$ and Dimitrios Kontogiannatos ${ }^{1}$
}

${ }^{1}$ School of Food, Biotechnology and Development, Department of Biotechnology, Laboratory of Molecular Biology, Agricultural University of Athens, Iera Odos 75, 11855, Athens, Greece.

Received: February 10, 2018; Accepted: March 27, 2018; Published: April 4, 2018

*Corresponding author: Anna Kourti, School of Food, Biotechnology and Development, Department of Biotechnology, Laboratory of Molecular Biology, Agricultural University of Athens, Iera Odos 75, 11855, Athens, Greece. Tel/Fax: +30 2105294615; E-mail: akourti@aua.gr

\begin{abstract}
A In order to understand whether and how the circadian system is connected to photoperiodism, an important piece of information is whether clock genes products oscillate and how they react to a changing photoperiod. In the moth Sesamia nonagrioides, which undergoes a facultive diapause controlled by photoperiod, we isolated the clock gene cryptochrome1 (cry1), named Sncry1. Sncry1 consists of 1762 bp encoding a polypeptide of 528 amino acid residues. SnCRY1 presented two characteristic conserved domains: the DNA photolyase and the Flavin-Adenine Dinucleotide (FAD) binding domain, which been demonstrated to be sufficient for light detection and phototransduction in Drosophila. SnCRY1 had significant homology with the CRY1 sequences identified from other insects. We also investigated the expression patterns of Sncry1 in brain of larvae growing under long-day 16L: 8D (LD), constant darkness (DD) and short-day 10L: 14D (SD) conditions using qRT-PCR assays. The mRNAs of Sncry1 expression was rhythmic in LD, DD and SD cycles. Sncry1 abundance tended to decrease during the day and then increase in the night. It is remarkable that the photoperiodic conditions affected the expression patterns and/or amplitudes of circadian clock gene Sncry1. Our data indicate that this gene may be associated with diapause in $S$. nonagrioides, because under SD (diapause conditions) the photoperiodic signal altered mRNA accumulation.
\end{abstract}

Keywords: Circadian clock; Cryptochrome; Photoperiodism; Diapause; Sesamia nonagrioides;

\section{Introduction}

There are two major rhythms of the biosphere, a daily cycle of night and day, and an annual seasonal cycle marked by changes in day and night length. The daily cycle is traced by an internal circadian clock that rules a large array of daily biochemical and physiological responses, while the seasonal cycle motivates photoperiodic responses that can be critical to survival, as in the case of insect diapause [1]. Circadian $(\cong 24 \mathrm{~h}$ ) clock is a core molecular mechanism that allows organisms to forestall daily environmental changes and adapt the timing of behaviors to maximize their efficacy. The basic circadian clock of insects functions as a light-delicate molecular oscillator, including a light-sensitive protein known as CRYPTOCHROME (CRY) and various feedback loops with positively and negatively substitute elements. The circadian clock in Drosophila is designed by cooperating molecular feedback loops containing of central clock and linked genes [2]. The expression of the two clock genes, period (per) and timeless (tim), is controlled by transcriptional activators, encoded by Clock ( $\mathrm{Clk})$ and cycle $(\mathrm{cyc})$. This initiation leads to periodic growth in the levels of per and tim mRNA tailed by the increase of PER and TIM proteins in cell nuclei, where PER actions as a repressor of CLK/CYC leading to overthrow of per and tim transcription. The photoreceptive CRY protein, encoded by the cryptochrome gene, intermediates the degradation of TIM, coordinating the clock to LD cycles. On contact to light, Drosophilatype cryptochrome (dCRY) encourages fast degradation of TIM that reduces PER unbalanced. PER is ultimately degraded, releasing the reserve of transcription [3]. Animal CRY proteins are phylogenetically separated into two groups: one comprises Drosophila-type CRY (CRY1) and the other contains all the vertebrate CRY (CRY2). Owing to ancestral gene duplication, there may be two cryptochrome genes in any certain insect species -cry1 and cry2 [4].

Photoperiodism, a reaction to the size of the light or dark period in a day, has been recognized in many insects, and controls diapause, seasonal morphs, growth rate, migration strategy, and a variability of related physiological states [5]. Diapause is a programmed stopped state of development that permits insects and other arthropods to continue opposing seasonal conditions either by becoming resting locally, or wandering to a more advantageous environment [6].

In order to comprehend whether and how the circadian system is associated to photoperiodism, a significant piece of evidence is whether clock genes products oscillate and how they respond to a varying photoperiod [7-9]. The connection of circadian clock genes in diapause initiation has been debated [1, 10-12].

The Mediterranean corn stalk borer, Sesamia nonagrioides (MCSB) undergoes facultative diapause and photoperiod rules the 
beginning of larval diapause. When diapause has been induced, larvae persist to grow and molt without pupating and up to six additional instars, elsewhere the normal five or six have been noticed, when larvae are continuous in diapausing conditions [11]. To accomplish indications to the link among the molecular mechanism of circadian and photoperiod clocks, in recent works we studied the expression of the clock genes period, timeless and cycle and the results suggested that transcriptional regulations of these clock genes act in the diapause programming in MCSB $[11,12]$. In the current study, we examined the contribution of cryptochrome1 (cry1) gene in the circadian rhythm and in the photoperiodic regulation of diapause in $S$. nonagrioides.

\section{Methods}

\section{Biological material and experimental schedules}

An established laboratory colony of $S$. nonagrioides, derived from field-collected larvae in Kopais (Latitude 380 14', Central Greece) was used as experimental biological material. Larvae were reared on artificial diet and were grown under photoperiods of $16 \mathrm{~h}$ light (L): $8 \mathrm{~h}$ dark (D) at $25 \pm 1^{\circ} \mathrm{C}$ and $65 \%$ relative humidity [11]. Photoperiods of 16L: 8D and continuous dark (DD) were used to encourage normal development while photoperiods of 10L: 14D were used to encourage diapause conditions. For quantitative real-time RT-PCR analyses (see below), larvae were reared until the 5th instar for long-day (16L: 8D) and constant darkness (DD) conditions while for short day conditions (10L: 14D) were reared until the 8th instar (day 55). Samples were collected every 3 hours starting at the zeitgeber time (ZT) 0 or circadian time (CT) 0 . The zeitgeber time (ZT) 0 was the time for the beginning of the light phase to which the phase associations were linked under two (long- and short- day) photoperiods while the circadian time (CT) 0 corresponded to the time schedule "lights-on" and circadian time (CT) 12 to the time schedule "lights-off" in DD conditions.

\section{cDNA synthesis, cloning and sequencing}

Total RNA was isolated according to the supplier's instructions from larvae using TRIzol $\AA$ reagent (Gibco BRL, Carlsbad, CA, USA). RNA concentration was estimated using a spectrophotometer (Spectronic model 21D, Carlsbad, CA, USA). Genomic DNA was removed using DNAse I (Invitrogen, Carlsbad, CA, USA). Partial clones of cryptochrome1 (cry1) from S. nonagrioides were isolated by RT-PCR from an RNA pool of nondiapausing larvae (5th instar). Total RNA was incubated with RNase-free DNAse I (Promega, Southampton, UK). Two $\mu$ g were used as template for the synthesis of the first strand cDNA synthesis with Superscript TM II RNase H-Reverse Transcriptase (Invitrogen). Degenerate primers were designed using conserved sequences from several Lepidoptera (Table 1). cDNA was used as template together with $200 \mu \mathrm{M}$ of each dNTP, 20 pmol of each primer, $2 \mathrm{U}$ of DNA polymerase (Expand-High Fidelity, Roche, Mannheim, Germany) and amplification was achieved in a thermal cycle (Model PTC200, M.J. Research, Waltham, MA), using a denaturation step (94 ${ }^{\circ} \mathrm{C}$ for $2 \mathrm{~min}$ ), and 35 cycles were run each with $94^{\circ} \mathrm{C}$ for $30 \mathrm{sec}, 55$
${ }^{\circ} \mathrm{C}$ for $30 \mathrm{sec}$, and $74{ }^{\circ} \mathrm{C}$ for $45 \mathrm{sec}$. PCR amplified fragment sizes approximately 800 bp were gel extracted and sequenced. The $3^{\prime}$ - and $5^{\prime}$-ends of the cDNA fragment were amplified according to Frohman [13]. For $3^{\prime}$-RACE, the first-strand cDNA was primed off with the T17XhO primer. Based on sequence information of the cDNA, two specific forward primers were designed: Cry Forward 1 and Cry Forward 2. PCR conditions were: $30 \mathrm{~s}$ at $94^{\circ} \mathrm{C}$, $1 \mathrm{~min}$ at $57^{\circ} \mathrm{C}$ and $2 \mathrm{~min}$ at $72{ }^{\circ} \mathrm{C}$, for 30 cycles followed by a final extension at $72{ }^{\circ} \mathrm{C}$ for $7 \mathrm{~min}$. For $5^{\prime}$-RACE, the synthesis of the first-strand cDNA was accomplished using the reverse specific primer Cry Reverse 1, was dA-tailed and amplified with the nested primer Cry Reverse 2 followed by the primer Cry Reverse 3 in combination with T17XHO. PCR conditions were: $30 \mathrm{~s}$ at $94{ }^{\circ} \mathrm{C}$, $1 \mathrm{~min}$ at $55^{\circ} \mathrm{C}$ and $2 \mathrm{~min}$ at $72^{\circ} \mathrm{C},(30$ cycles) followed by a final extension of $72{ }^{\circ} \mathrm{C}$ for $7 \mathrm{~min}$. Sequences for Sncry 1 are deposited on EMBL/GENBANK as the accession number DQ243705.

\section{Sequencing and Phylogenetic analysis}

The amino acid sequence of Sncry1 was predicted using the DNAman v.5.2.2 software (Lynnon Biosoft, Quebec, Canada) and was submitted to the NCBI website (http://www.ncbi.nlm. nih.gov). The $\mathrm{pI}$ and MW were computed using the Compute $\mathrm{pI} /$ MW software (http://www.expasy.ch/tools/pi tool.html). The post-translational modification sites were predicted using the Prosite Scan software (http://npsa-pbil.ibcp.fr/cgi-bin/npsa automat.pl?page=npsa prosite.html), while secondary structures were determined using the SOPMA software (http://npsa-pbil. ibcp.fr/cgi-bin/npsa automat.pl?page=/NPSA/npsa sopma. $\underline{\mathrm{html}}$ ). The 3D structures were constructed using the Swiss Model Workspace (http://swissmodel.expasy.org). Phylogenetic trees and P-Distances were constructed using the neighbourjoining method with the MEGA5 software $[14,15]$. To construct the phylogenetic tree, known sequences of predicted CRY proteins from other insect species were attained from GenBank. Bootstrapping was used to estimate the reliability of phylogenetic reconstructions (1000 replicates) [16]. Abbreviations and accession numbers of genes in the phylogenetic trees are listed in the figure legends.

\section{Quantitative Real-Time PCR (qRT-PCR)}

Larvae heads were dissected and used to isolate total RNA. Larvae were reared under long-day, constant dark or short-day conditions. For quantitative real time RT-PCR (qRT-PC), we used specific primers (Table 1 ) and the incorporation of the fluorescent dye SYBR Green Brilliant (Stratagene, LA Jolla, CA, USA) into double-stranded PCR products was estimated. Plasmids were constructed into pGEM T-easy vector (Promega, Madison, WI, USA) by inserting a $\sim 200 \mathrm{bp}$ fragment from the coding region of each gene amplified from individual cDNA with gene specific primers Cry RT forward and Cry RT reverse (Table 1). We designed real-time PCR primers from the Sncry1 sequences at 3'UTR (Table 1). The recombinant plasmids were used as template to produce standard curves using a Stratagene MX3005PTM Real Time PCR system (Stratagene) at concentrations ranging from 1 


\begin{tabular}{|c|c|}
\hline Oligonucleotides & Sequence $\left(5^{\prime} \rightarrow 3^{\prime}\right)$ \\
\hline \multicolumn{2}{|l|}{ Degenerate PCR } \\
\hline Cry forward: & $\begin{array}{l}\text { C T ( C / G ) A C C T A C C A ( A / G ) } \\
\text { ATGTTC(C/T)TGCA }\end{array}$ \\
\hline Cry reverse: & $\begin{array}{l}\mathrm{A}(\mathrm{C} / \mathrm{T}) \operatorname{ACCCACATCCA}(\mathrm{A} / \mathrm{G}) \mathrm{TT}(\mathrm{A} / \mathrm{G}) \\
\mathrm{CC}(\mathrm{A} / \mathrm{G}) \mathrm{GC}\end{array}$ \\
\hline \multicolumn{2}{|l|}{ RACE PCR } \\
\hline 3' - Cry Forward 1: & AACTACGGACAGATGAGTGGCAA \\
\hline 3' - Cry Forward 2: & AACACTCTGGCTGTCGTGGGAA \\
\hline 5' - Cry Reverse 1: & TAAGGAAGAACGCCACAGTGTTGC \\
\hline $\begin{array}{l}\text { 5' - Cry Reverse 2: } \\
\text { 5' - Cry Reverse 3: }\end{array}$ & $\begin{array}{l}\text { TGATGCACCTCACGGAATAGGTCC } \\
\text { ATCTGTTTAAGCGCAGTGGTCTC }\end{array}$ \\
\hline \multicolumn{2}{|c|}{ Real-time PCR assays } \\
\hline Cry RT forward: & AGAGCGTGCGTCCTGTATAATAG \\
\hline Cry RT reverse: & CCGTAAGTCATTACTCGTTGAGC \\
\hline Sn RT-TubF & GAGCAGTTCACCGCTATGTTC \\
\hline Sn RT-TubR & GGTGTGAGTGCTTTAGTTGTCC \\
\hline
\end{tabular}

ng to $10 \mathrm{fg}$. The amplification cycle was: $95^{\circ} \mathrm{C}$ for $10 \mathrm{~s}, 56^{\circ} \mathrm{C}$ for $30 \mathrm{~s}$ and $72{ }^{\circ} \mathrm{C}$ for $30 \mathrm{~s}$ ( 35 cycles) using 5 pmol of each primer. The mRNA levels was normalized with $S$. nonagrioides $\beta$-tubulin (GENBANK accession no. DQ147771) gene and quantified in the same manner. Data are expressed as means \pm SEM of 4 independent biological replicates and 3 technical replicates $(\mathrm{N}=$ 12/time point) and estimated by ANOVA and the Tukey's post hoc test using the IBM SPSS software (IBM Analytics).

\section{Results}

\section{Sequence of Sncry1}

The full-length nucleotide sequence of cry1 cDNA of $S$. nonagrioides (Sncry1, GenBank Accession no. DQ243705) consists of $1762 \mathrm{bp}$. Conceptual translation of the cDNA sequence yields a 5' untranslated region (UTR) of $102 \mathrm{bp}$, a 3'-terminal UTR of 76 bp with a poly (A) tail, and an open reading frame (ORF) of 1584 bp, encoding a polypeptide of 528 amino acid residues (Figure 1). The molecular mass of the deduced SnCRY1 protein was predicted to be $59.6 \mathrm{kDa}$, and the calculated isoelectric point (pI) was 8.33. Comparing the deduced amino acid sequence of SnCRY1 (NCBI, BLAST) revealed that SnCRY1 had significant homology with the CRY1 sequences identified from other insects, such as Mamestra bracicae (AY947639, 86\%), Helicoverpa armigera (AEX49898, 86\% identity), Mythimna separate (AFR54426, 86\% identity), Antheraea pernyi (AAK11644.1, 80\% identity), Bombyx mori (NP_001182628.1, 75\% identity). SnCRY1 presented two characteristic conserved domains: the DNA photolyase and the Flavin-Adenine Dinucleotide (FAD) binding domain (Figure 1), which been demonstrated to be sufficient for light detection and phototransduction in Drosophila [17]. Multiple alignments (Figure 2) revealed that the N-terminus of the proteins showed the highest conservation in analyzed SnCRY1 sequences, whereas the C-terminal had lower levels of conservation. The conserved regions of SnCRY1 and contained DNA photolyase domains and FAD binding domains, which were highly conserved among the different CRY1s (Figure 2).

The secondary structures were constructed by SOPMA, and the rates determined were $37.31 \%$ of alpha helix, $9.85 \%$ of beta turn, $14.58 \%$ of extended strand and $38.26 \%$ of random coil. The crystallographic structures of CRY1 are very important for their functions. The structures of SnCRY1 (Figure 3) was predicted, based on the three-dimensional structure of Drosophila melanogaster (6-4) photolyase 3cvvA 224 (2.10 A). Monomer structure comparison of 4 jzy.1.A. template (D. melanogaster), show Seq Identity $52.67 \%$ and Description as Cryptochrome 1. Also identifired Ligads: $1 \mathrm{XFAD}$ and matching prediction $1 \mathrm{x}$ FLAVIN-ADENINE DINUCLEOTIDE. Ligand 1 in contact with: Chain A : R229, S257, L258, S259, L262, Q300, L301, W303, R304, F307, W364, L365, H367, R370, N371, F393, D399, A400, D401, V404, C405, N408, W409, F523.

A phylogenic tree (Figure 4) based on the CRYs from different insects was constructed using the neighbor-joining method. Phylogenetic analysis showed that CRYs in insects could be classified into two cluster CRY1 and CRY2. The result of the phylogenetic analysis agreed with the structure and distribution of these CRYs. The SnCRY1 belongs to the CRY1 cluster. Moreover, the phylogenetic tree demonstrated that $S$. nonagrioides had a shorter genetic distance to Lepidopteran species than other insects, which was consistent with the traditional taxology.

\section{Expression patterns of Sncry1 under different photoperiods}

To determine if Sncry1 transcripts oscillate, we examined the levels of gene mRNAs in the head of larvae under 16L: 8D (long day, LD), constant darkness (DD) and 10L:14D (short day, SD) by performing Real-Time PCR assays and showed that the expression was rhythmic. Under 16L: 8D conditions a clear diel rhythm of cry1 mRNA levels was detected (Figure 5A): the expression of Sncry1 mRNA appeared to cycle with a peak during the night (ANOVA, p<0.01). The peak value at ZT 21 ( $5 \mathrm{~h}$ after onset of scotophase) was about 7 times higher than the trough level (at ZT 15) and the difference was statistically significant (Tukey's test, $\mathrm{p}<0.01$ ). The oscillation of Sncry1 mRNA under LD also appeared in three days observations (data not shown). The rhythmic cry1 mRNA expression persisted in DD (Figure 5B) with a peak at late subjective night (CT21; ANOVA, $p<0.01$ ), and the peak was about 5-fold of the trough level. Under 10L: 14D conditions, a clear diel rhythm of Sncry1 mRNA levels was also with two peaks: one during the day (at ZT3) and the other during the night (at ZT12) (Figure 5C). The oscillation of Sncry1 mRNA under SD photoperiod was quite different than LD: the peak value was shifted to early in the scotophase at ZT 12 (2 h after onset of the scotophase), and the peak value was about 4 times higher than the trough level. The oscillation of Sncry1 mRNA under SD appeared also in three days observations (data not shown). By 


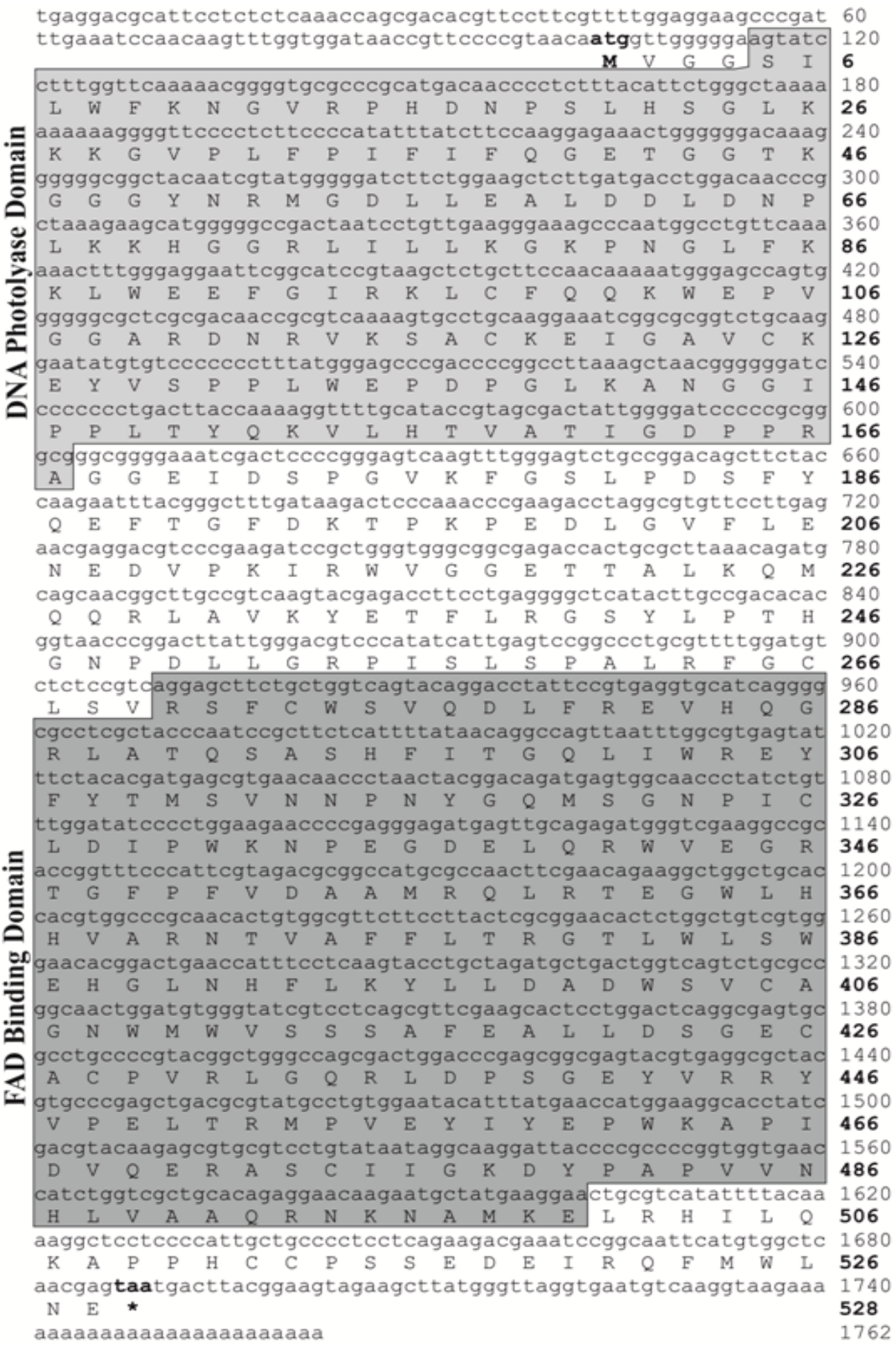

Figure 1: The nucleotide and deduced amino acid sequences of Sncry1. The positions of the nucleotides and amino acids were indicated in the right margin. The termination codon was marked with a star. Faction motif of cryptochrome correspondence of D. melanogasterare marked: DNA Photolyase Domain; FAD (Flavin-Adenine Dinucleotide) binding domain of DNA photolyase. 


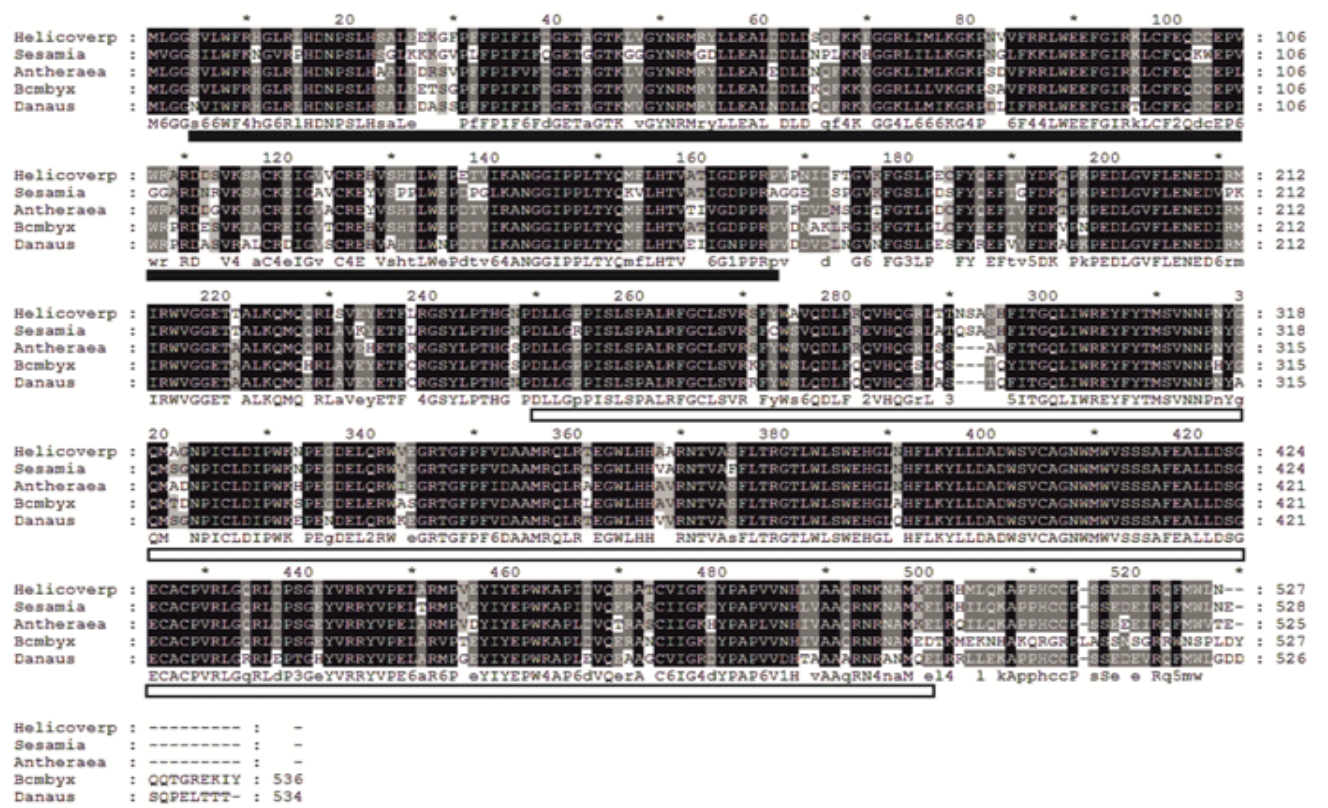

Figure 2: Alignment of the SnCRY1 amino acid sequence with sequences of other lepidopteran CRY1s: numbers on the right side of the alignment indicate the position of residues in the sequence of each protein. Conserved residues of the five sequences are shaded in black. The DNA-photolyase domain was indicated by black bar under the alignment (position 5-167 in S. nonagrioides) while the white bar indicates the FAD binding domain (position 250-500 in S. nonagrioides). The GenBank accession numbers: Antheraea: A. pernyi (AAK11644.1); Bombyx: B. mori (NP_001182628.1); Danaus: D. plexippus (AAX58599.1); Helicoverpa: H. armigera: (ADN94464.1); Sesamia: S. nonagrioides (ABB52818.2).

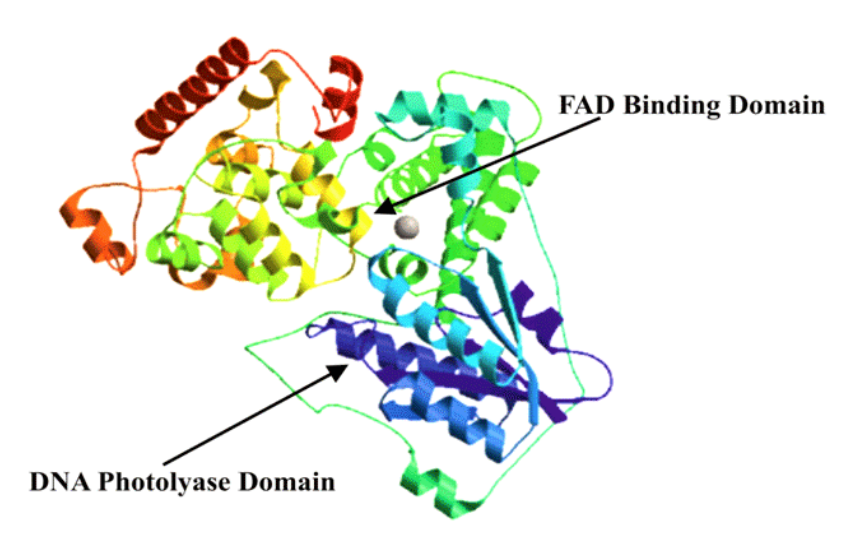

Figure 3: Molecular modeling into the structural feature of S. nonagrioides CRY1.

comparing the ratios between the peak: trough levels under short and long day conditions, it seems that the amplitude of the Sncry 1 mRNA expression was weaker at SD than at LD.

\section{Discussion}

In the current study, we reported on the structure and expression profile of the clock gene cryptochrome1 (Sncry1) in the moth Mediterranean corn stalk borer (MCSB), S. nonagrioides. This insect undergoes a facultative diapause characterized by prolonged larval duration in reaction to short-day conditions. The nucleotide and amino acid sequences of Sncry1 (Figure 1)

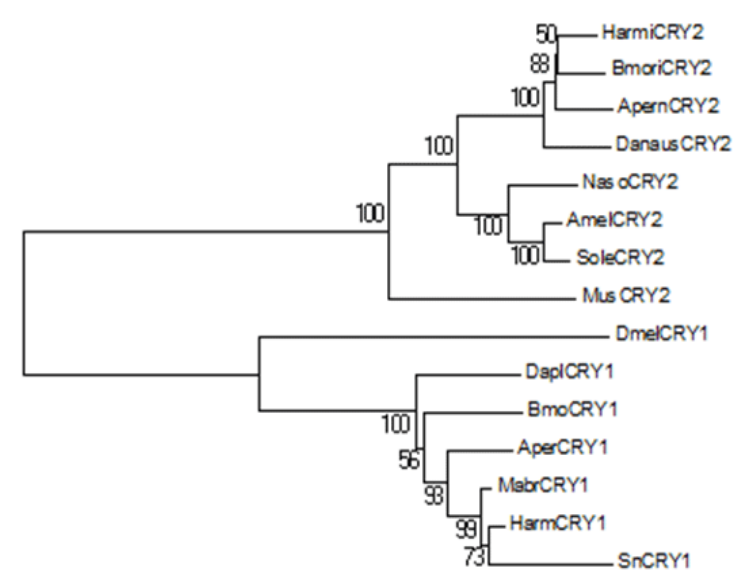

01

Figure 4: Phylogenetic analysis based on CRY1 and CRY2 amino acid sequences. The numbers at the nodes indicated the bootstrap. The GenBank accession numbers used: DmelCRY1(D. melanogaster: NP_732407.1); BmoCRY1 (B. mory: NP_001182628.1); AperCRY1 (A. pernyi: AAK11644.1); MbrCRY1 (M. brassicae: AAY23345.1); Dapl-CRY1 (D. plexippus: AAX58599.1); HarmCRY1 (H. armigera: ADN94464.1); SnCRY1(S. nonagrioides: ABB52818.2); HamCRY2 (H. armigera: ADN94465.1); BmorCRY2 (B. mori: ADM86935.1); AperCRY2 (A. pernyi: AB038435.1), DanausCRY2 (D. plexippus: ABA62409.1); NasCRY2 (Nasonia vitripennis: XP_001606405.2); AmelCRY2 (A. mellifera AB038437.1); SoleCRY2 (Solenopsis invicta: JX948389); MusCRY2 (Mus musculus: NP031797). 
A.

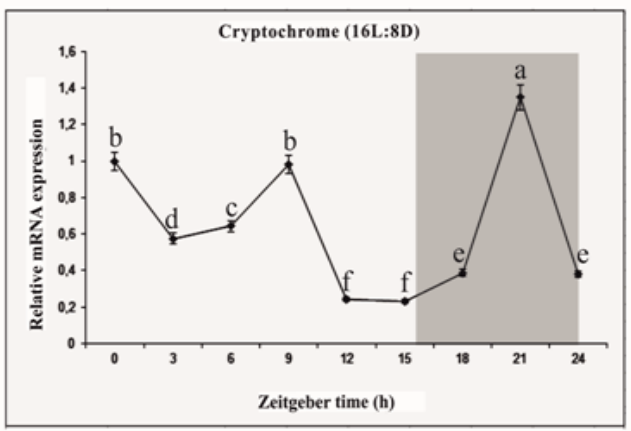

C.

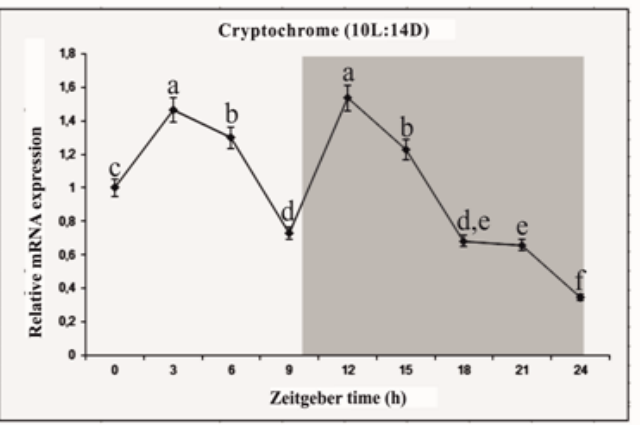

B.

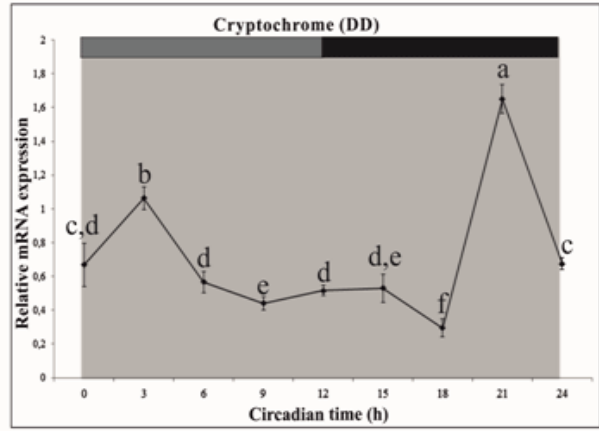

Figure 5: Daily and circadian expression patterns of Sncry1 mRNA in the heads of 5th instar MCSB larvae in: A: 16L: 8D (lights on at ZT0 and lights off ZT16), B: constant dark DD (lights off) and C: 10L: D14D (lights on at ZT0 and lights off ZT10). Larvae were entrained in light-dark cycles for 25 days and the tissues were collected at ZT 0, ZT3, ZT6, ZT9, ZT12, ZT15, ZT18, ZT21, and ZT24. Shaded area shows the scotophase. Bars represent Mean \pm SEM of 4 independent biological replicates plus 3 technical replicates ( $\mathrm{N}=12 /$ time point). Letters indicate statistical significance (One way ANOVA followed by the Tukey's post hoc test).

indicated high similarities to previous identified CRY1s. SnCRY1 contains a C-terminal domain and the photolyase homology domain consists of a DNA-Photolyase domain and a FAD binding7 domain $[18,19]$. Busza et al. establish that the photolyase homology domain was for light discovery and phototransduction, whereas C-terminal domain regulated CRY solidity, CRY-TIM contact and circadian photosensitivity [17]. The structural protection of the DNA photolyase and the FAD binding domains among the different CRYs might be the suggestion of common mechanistic features, principally in photoreception [19]. Many organisms display circadian rhythms generated by the circadian clock; the photoreceptor is essential for the clock to coordinate the light-dark cycles and photic entrainment depends on the function of CRY. Phylogenetic analyses appearance at least two rounds of gene duplication at the base of the metazoan radiation, as well as several losses, offered growth to two cryptochrome (cry) gene families in insects, a Drosophila-like cry1 gene family and a vertebrate-like cry2 family. These genes are similar in their nucleotide and amino acid sequences, but paralogous and apparently different by phylogenetic analyses [4]. The CRY1 acts as a blue-light photoreceptor for photic entrainment; the CRY2 roles as a major transcriptional repressor but not as a circadian photoreceptor $[20,21]$. The predicted crystallographic structures of SnCRY1 (Figure 3) presented that it is a blue-light photoreceptor. The phylogenetic tree in our study (Figure 4) discovered that SnCRY1 belongs to the cry1 family. As established by the phylogenetic tree, the CRY1 sequence of $S$. nonagrioides was closer to those of Helicoverpa armigera and Mamestra bracicae than those of non-Lepidopteran insects. This result adapted well to the traditional classes of these species.

Expression analysis of Sncry1 using qRT-PCR was rhythmic in LD, DD and SD cycles in the larvae head of MCSB (Figure 5). Cycling of Sncry1 under DD condition (Figure 5B) insisted with phases and amplitudes similar to that observed in LD condition, demonstrating that it was under circadian regulation. In Drosophila, cry mRNA cycle under a light-dark cycle (LD), with high levels in light and low levels in the dark. This cycling persists in continuous darkness (DD), but with reduced amplitude [22]. Unlike, in MCSB to LD condition, Sncry1 abundance have a tendency to decrease during the day, then increase in the night. An analogous result is observed in $H$. armigera moths under LD conditions for Hacry2, whereas Hacry1 abundance tended to increase during the day and then decrease in the night [23]. The dCRY1 structure reveal that the tail residue Cys523 plays key roles in the dCRY photoreaction [24]. The 3D structure of SnCRY1 (Figure 3) show similarities with dCRY1 in this location but details of the role that photoreceptors play in the photic entrainment of MCSB need to be additional investigated. 
In our study (Figure 5) we showed that the Sncry1 mRNA peaked in the mid night comparable to Snper [11]. The discovery of the existence of two cry genes in moths and butterflies suggested that the clockwork mechanism of Lepidopteran insects differ from that of D. melanogaster [25]. In species where cry2 is existing, its oscillation matches that of per, as revealed in our study, with a trough in the light phase and a highest in the dark phase [26-28]. In Drosophila, PER is the key negative regulator of clock function, but until now, in all insects where cry2 is present and irrespective of cry1's presence, CRY2 shows this role [29]. In a previous work we found that the chronological pattern of gene expression in MCSB brain is amazingly distinct from that of Drosophila [12]. In the MCSB, Sncyc and Snper mRNA levels oscillate with a similar phase, but in Drosophila they are in antiphase $[30,31]$. The ancestral circadian clock maybe involved CYC as the positive-acting transcriptional activator, CRY1 as the main photoreceptor, and CRY2 as the light in sensitive, negative acting transcriptional regulator [25]. Drosophila expresses CRY1 only, while some insects, like mosquitos and butterflies, express both CRY1 and CRY2 [32]. The honeybee Apis mellifera and the beetle Tribolium castaneum contain only CRY2 [4, 32]. This proposes two significant options. First, the core oscillator in insects has itself evolved such that at least three kinds of clocks exist, those having only CRY1 as in Drosophila, those enclosing CRY1 and CRY2 as in monarch and mosquito, and those containing CRY2 only as in beetle and honeybee. Second, in insects enclosing only CRY2, the cryptochrome may service dual functions, as both a transcriptional repressor and a photoreceptor [25]. In a previous work we found that the cycle gene in MCSB show interesting changes related to Drosophila, proposing that this species is a remarkable new model to study the molecular control of insect biological clocks [12]. We speculate that in insects maybe there is another possibility, such as in MCSB, where it seems that CRY1 alone, could evolve dual functions as transcriptional repressor and a photoreceptor. An interesting observation is that Snper, Sntim, Sncyc and Sncry1 synchronously peaked at midnight, revealing

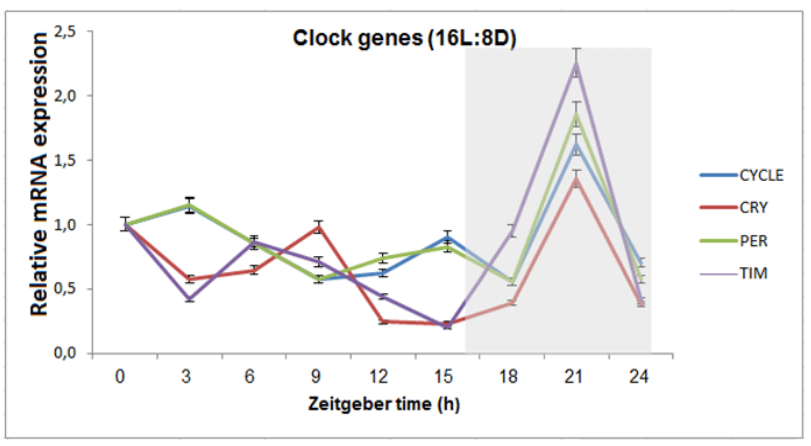

Figure 3: MDaily and circadian expression patterns of Snper, Sntim, Sncyc and Sncry1 mRNA in the heads of 5th instar MCSB larvae in: A: 16L: 8D (Lights on at ZT0 and lights off ZT16). Larvae were entrained in light-dark cycles for 25 days and the tissues were collected at ZT 0, ZT3, ZT6, ZT9, ZT12, ZT15, ZT18, ZT21, and ZT24. Shaded area shows the scotophase. Data of Snper, Sntim and Sncyc are from [11, 12]. that MCSB has a distinct circadian cycle when compared to Drosophila (Figure 6). In Drosophila, cry expression is regulated at the transcriptional level by the clocks and at the translational and posttranslational level by the light [22]. In our case light and clock regulates the transcriptional regulation of Sncry1 gene (Figure 6). The transcript accumulation profile suggests that Snper, Sntim, Sncyc and Sncry1 genes are coordinately regulated by light. While in Drosophila, CRY intermediates the degradation of TIM, in MCSB the coordinated expression of Sntim and Sncry 1 suggests that cry activation is regulated at translational and/or posttranslational level [3]. This conjecture is still unclear and needs further investigation.

In our study the expression of the circadian gene Sncry 1 was directly related to diapause-inducing photoperiod. The Sncry1 mRNA oscillation exhibited a peak $5 \mathrm{~h}$ after onset of the scotophase under long days and shifted $2 \mathrm{~h}$ after onset of the scotophase under short days. Sncry1 showed association with diapause in S. nonagrioides, since under 10L: 14D (diapausing conditions) the photoperiodic signal produced alteration of mRNAs. Under short-day conditions, higher levels of Sncry1 where detected in larval heads than those reared under longday conditions. In addition, a difference exhibited in the Sncry 1 mRNA oscillation after onset of the scotophase (Figure 5C). Possibly, the alteration of mRNA levels of Sncry1, is necessary for the expression of diapause in $S$. nonagrioides. The connection of circadian clock genes in diapause induction has been discussed and some circadian clock genes have been recommended to be linked with diapause in insects [7, 6, 11, 12, 32]. In the parasitic wasp, Nasonia vitripennis, the circadian oscillation of per and cry mRNAs in the heads of Nasonia females, also kept under short and long photoperiods [33]. It is generally accepted that a circadian clock is involved in the photoperiodic response and photoperiods often modulate the circadian parameters and the waveform of the clock $[34,35]$. Thus, if the circadian clock were involved, the photoperiodically moderated waveform would have some roles in the photoperiodic time measurement [36]. Interestingly, the $S$. nonagrioides showed strong modulated waveform in the clock gene Sncry1 expression and a drastic difference in the expression level of Sncry1 was observed between different photoperiodic conditions (LD and SD). At present, few studies have studied cry expression during different photoperiods. Here, our results show that transcriptional regulation of Sncry1 acts in diapause programming in MCSB and may be essential for daily rhythms and photoperiodic diapause.

In order to realize whether and in what way the circadian system is associated to photoperiodism, a significant part of information is whether clock genes products fluctuate and how they respond to a changing photoperiod. Our data on the clock genes Snper, Sntim, Sncyc and Sncry1 revealed that in the MCSB the expression patterns of these oscillate and affected by photoperiod $[11,12]$. Since our experiments were done under LD, DD and SD conditions, the data reflect how the molecular clock adapts to photoperiodic changes. Our results show that 
transcriptional regulation of these four clock genes maybe play important roles in the diapause programming in MCSB.

\section{Acknowledgment}

We are grateful to Theodoros Gkouvitsas for advice and technical assistance.

\section{References}

1. Bradshaw WE and Holzapfel CM. What season is it anyway? Circadian tracking vs. Photoperiodic anticipation in insects. J Biol Rhythms. 2010;25(3):155-165. Doi: 10.1177/0748730410365656.

2. Hardin PE. The circadian timekeeping system of Drosophila. Curr Biol. 2005;15(17):R714-722.

3. Allada R and Chung BY. Circadian organization of behavior and physiology in Drosophila. Annu Rev Physiol. 2010;72:605-624. Doi: 10.1146/annurev-physiol-021909-135815.

4. Rubin EB, Shemesh Y, Cohen M, Elgavish S, Robertson HM and Bloch G. Molecular and phylogenetic analyses reveal mammalianlike clockwork in the honey bee (Apis mellifera) and shed new light on the molecular evolution of the circadian clock. Genome Res. 2006;16(11):1352-1365.

5. Saunders DS. Photoperiodism in insects: migration and diapause responses. DL. Denlinger (Ed.), Photoperiodism: The Biological Calendar, Oxford University Press. 2010:218-257.

6. Meuti ME and Denlinger DL. Evolutionary Links between Circadian Clocks and Photoperiodic Diapause in Insects. Integrative Comp Biol. 2013;53(1):131-143. Doi: 10.1093/icb/ict023.

7. Koštál V. Insect photoperiodic calendar and circadian clock: Independence, cooperation, or unity? J Insect Physiol. 2011;57(5):538556. Doi: 10.1016/j.jinsphys.2010.10.006.

8. Saunders DS and Bertossa RC. Deciphering time measurement: the role of circadian "clock" genes and formal experimentation in insect photoperiodism. J.Insect Physiol. 2011;57(5):557-566. Doi: 10.1016/j.jinsphys.2011.01.013.

9. Goto SG. Roles of circadian clock genes in insect photoperiodism. Entomol Sci. 2013;16(1):1-16.

10. Danks HV. How similar are daily and seasonal biological clocks? J.Insect Physiology. 2005;51(6):609-619.

11. Kontogiannatos D, Gkouvitsas $\mathrm{T}$ and Kourti A. The expression patterns of the clock genes period and timeless are affected by photoperiod in the Mediterranean corn stalk borer, Sesamia nonagrioides. Arch Insect Biochem Physiol. 2016;94(1):1-15.

12. Kontogiannatos D, Gkouvitsas $\mathrm{T}$ and Kourti A. The expression of the clock gene cycle has rhythmic pattern and is affected by photoperiod in the moth Sesamia nonagrioides. Compar Biochem Physiol Biochem Mol Biol. 2017;208-209:1-6. Doi: 10.1016/j.cbpb.2017.03.003.

13. Frohman MA. RACE: Rapid amplification of cDNA ends. In PCR Protocols: A Guide to Methods and Applications. Eds. M.A. Innis, D.H. Gelfand, J.J. Sninsky and T.J. White. Academic Press, London; 1990:2838.

14. Saitou N and Nei M. The neighbor-joining method: a new method for reconstructing phylogenetic trees. Mol. Biol. Evol. 1987; 4(4):406-425.

15. Tamura K, Peterson D, Peterson N, Stecher G, Nei M and Kumar S. MEGA5: Molecular Evolutionary Genetics Analysis Using Maximum
Likelihood, Evolutionary Distance, and Maximum Parsimony Methods. Mol Biol Evol. 2011;28(10):2731-2739. Doi: 10.1093/molbev/ msr121.

16. Felsenstein J. PHYLIP (Phylogeny Inference Package) version 3.6alpha. Distributed by the author. Department of Genetics, University of Washington, Seattle, 2001.

17. Busza A, Emery-Le M, Rosbash M and Emery P. Roles of the two Drosophila CRYPTOCHROME structural domains in circadian photoreception. Science. 2004;304(5676):1503-1506.

18. Cashmore AR. Cryptochromes: enabling plants and animals to determine circadian time. Cell. 2003;114(5): 537-543.

19. Merlin C, François MC, Queguiner I, Mabïèche-Coisné M and JacquinJoly E. Evidence for a putative antennal clock in Mamestra brassicae: molecular cloning and characterization of two clock genes -period and cryptochrome-in antennae. Insect Molecular Biology. 2006;15(2):137145.

20.Zhu H, Sauman I, Yuan Q, Casselman A, Emery-Le M, Emery P and Reppert SM. Cryptochromes define a novel circadian clock mechanism in monarch butterflies that may underlie sun compass navigation. PLoS Biol. 2008; 6(1): e4. Doi: 10.1371/journal.pbio.0060004.

21. Tomioka $\mathrm{K}$ and Matsumoto A. A comparative view of insect circadian clocks. Cel Mol Lif Scien. 2010;67(9):1397-1406. Doi: 10.1007/ s00018-009-0232-y.

22. Emery P, So WV, Kaneko M, Hall JC and Rosbash M. CRY, a Drosophila clock and light-regulated cryptochrome, is a major contributor to circadian rhythm resetting and photosensitivity. Cell. 1998;95(5):669679.

23. Yan S, Ni H, Li HT, Zhang J, Liu XX and Zhang QW. Molecular cloning, characterization, and mRNA expression of two cryptochrome genes in Helicoverpa armigera (Lepidoptera: Noctuidae). J Econ Entomol. 2013;106(1):450-462.

24. Czarna A, Berndt A, Singh HR, Grudziecki A, Ladurner AG and Timinszky G, et al. Structures of Drosophila cryptochrome and mouse cryptochrome1 provide insight into circadian function. Cell. 2013;153(6):1394-1405. Doi: 10.1016/j.cell.2013.05.011.

25.Yuan QD, Metterville AD, Briscoe $S$ and Reppert M. Insect cryptochromes: gene duplication and loss define diverse ways to construct insect circadian clocks. Mol Biol Evol. 2007;24(4):948-955.

26. Ingram KK, Kutowoi A, Wurm Y, Shoemaker D, Meier R and Bloch G. The molecular clockwork of the fire ant Solenopsis invicta. PLoS One. 2012;17(11):e45715. Doi: 10.1371/journal.pone.0045715.

27. Werckenthin A, Derst $C$ and Stengl M. Sequence and expression of per, tim1, and cry2 genes in the Madeira cockroach Rhyparobia maderae. J Biol Rhythm.2012;27(6):453-466. Doi:10.1177/0748730412462109.

28. Egan ES, Franklin TM, Hilderbrand-Chae MJ, McNeil GP, Roberts MA and Schroeder AJ, et al. An extraretinally expressed insect cryptochrome with similarity to the blue light photoreceptors of mammals and plants. J Neurosci. 1999;19(10):3665-3673.

29. Sandrelli F, Costa R, Kyriacou CP and Rosato E. Comparative analysis of circadian clock genes in insects. Insect Mol Biol. 2008; 17(5):447463. Doi: $10.1111 /$ j.1365-2583.2008.00832.x.

30.Glossop NRJ, Houl JH, Zheng H, Ng FS, Dudek SM and Hardin PE. VRILLE feeds back to control circadian transcription of Clock in the Drosophila circadian oscillator. Neuron. 2003;37(2):249-261. 
31. Zhu H, Yuan Q, Froy 0, Casselman A and Reppert SM. The two CRYs of the butterfly. Curr Biol. 2005;15(23):R953-R954.

32. Ikeno T, Tanaka S, Numata H and Goto SG. Photoperiodic diapause under the control of circadian clock genes in an insect. BMC Biol. 2010;8:116-125. Doi: 10.1186/1741-7007-8-116.

33. Bertossa RC, Zande L, Beukeboom LW and Beersma DGM. Phylogeny and oscillating expression of period and cryptochrome in short and long photoperiods suggest a conserved function in Nasonia vitripennis. Chronobiol. Int. 2014;31(6):749-760. Doi: 10.3109/07420528.2014.880451.
34. Saunders DS. Insect Clocks, 3rd ed. Elsevier Science, Amsterdam, 2002:560.

35. Koga M, Ushirogawa $\mathrm{H}$ and Tomioka K. Photoperiodic modulation of circadian rhythms in the cricket Gryllus bimaculatus. J Insect Physiol. 2005;51(6):681-690.

36. Sakamoto T, Urya 0 and Tomioka K. The clock gene period plays an essential role in photoperiodic control of nymphal development in the cricket Modicogryllus siamensis. J Biol Rhythms. 2009;24(5):379-390. Doi: $10.1177 / 0748730409341523$. 